\title{
Identification of Pemphigus Vulgaris Antigen Extracted from Normal Human Epidermis and Comparison with Pemphigus Foliaceus Antigen
}

Russell W. Eyre and John R. Stanley

Dermatology Branch, National Cancer Institute, National Institutes of Health, Bethesda, Maryland 20892

\begin{abstract}
Immunoprecipitations of cultured keratinocyte extracts have shown that pemphigus vulgaris (PV) sera bind a polypeptide of 210,000 mol wt with disulfide-linked chains of 130,000 and 85,000 mol wt. To identify proteins in normal human skin recognized by $P V$ antibodies, we performed immunoprecipitations of normal human epidermal extracts. All 22 PV sera tested immunoprecipitated a complex of polypeptides (PV complex) of $210,000,130,000$, and $85,000 \mathrm{~mol}$ wt, after reduction. One- and two-dimensional gel electrophoresis showed that the 130,000- and 85,000-mol-wt polypeptides of the PV antigen from both cultured keratinocytes and epidermis have identical charges and sizes. In addition to precipitating the PV complex, 14 of 22 PV sera also have antibodies to a calciumsensitive epitope on a different complex of polypeptides (PF complex) which has previously been shown to be precipitated by all pemphigus foliaceus (PF) sera. The PF complex consists of polypeptides of $260,000,160,000,110,000$, and $85,000 \mathrm{~mol}$ wt. Although the majority of PV sera also precipitate the PF complex, no PF sera precipitate the PV complex. Thus, PV and PF can be absolutely distinguished on a molecular level using the patients' autoantibodies. The PV and PF complexes, although distinct, have certain similarities. The 85,000-mol-wt polypeptide of each is identical. The $160,000-\mathrm{mol}$ wt-peptide of the PF complex and the 130,000 -mol-wt peptide of the PV complex have the same isoelectric point and both are capable of disulfide linkage to the 85,000 -mol-wt polypeptide. The PV and PF complexes are closely related and may prove important in cell adhesion.
\end{abstract}

\section{Introduction}

Pemphigus, an autoimmune blistering disease, exists as two major types (1). In pemphigus vulgaris (PV $)^{1}$ mucosal and skin blisters develop because epidermal cells come apart in a suprabasilar location through a process called acantholysis. In pemphigus foliaceus (PF), or superficial pemphigus, mucosal lesions are rare and skin blisters develop in a subcorneal loca-

Address correspondence to Dr. Stanley, Bldg. 10, Room 12N-238, National Institutes of Health, Bethesda, MD 20892.

Received for publication 25 June 1987 and in revised form 8 September 1987.

1. Abbreviations used in this paper: $\mathrm{PF}$, pemphigus foliaceus; PF complex, a complex of polypeptides of $260,000,160,000,110,000$, and 85,000 mol wt immunoprecipitated by PF sera; PV, pemphigus vulgaris; PV complex, a complex of polypeptides of $210,000130,000$, and 85,000 mol wt immunoprecipitated by PV sera; TBS, Tris-buffered saline.

The Journal of Clinical Investigation, Inc.

Volume 81, March 1988, 807-812 tion due to acantholysis of the superficial-most epidermal cells. Autoantibodies to the surface of keratinocytes throughout the epidermis are present in both types of pemphigus.

Recently, using an immunoprecipitation procedure with extracts of normal human epidermis, we have shown that all PF patients have antibodies to a complex of polypeptides with a calcium-sensitive epitope (2). This complex (PF complex) consists of polypeptides of 260,000, 160,000 (desmoglein I), 110,000 , and 85,000 apparent molecular weight. The 160,000-mol-wt peptide of the PF complex was shown to be desmoglein I, a core desmosomal glycoprotein $(2,3)$.

There has been conflicting data regarding the identity of the PV antigen extracted from normal human epithelia. From esophogeal mucosal extracts a 68,000 -mol-wt protein was identified using an immunofluorescence absorption technique (4). A similar technique was used to identify a 20,000- and a 25,000-mol-wt complex from saliva (5). More recently a 33,000-mol-wt protein has been identified by immunoblotting of human skin extracts (6). A 140,000-mol-wt protein has been identified by immunoblotting of bovine tongue, but not bovine skin, extracts (7).

In our laboratory we have been unable to identify the PV antigen by immunoblotting of normal human epidermal extracts. However, we have characterized the PV antigen synthesized by cultured human keratinocytes $(8,9)$. Immunoprecipitation of metabolically labeled cultured keratinocytes has indicated that all PV sera bind a 210,000-mol-wt polypeptide with disulfide-linked chains of 130,000 and $85,000^{2}$ mol wt (9). In addition, we have shown that the PV antigen from cultured keratinocytes no longer reacts with the antibody after the antigen has been denatured for immunoblotting experiments (9). With this finding in mind, we hypothesized that the PV antigen extracted from normal human skin might also, once denatured for immunoblotting, no longer bind (or bind very poorly to) the autoantibodies from PV patients. Thus, in this study, to identify PV antigen in human epidermis, we used a minimally denaturing extraction procedure and performed immunoprecipitation before denaturing the proteins for SDSPAGE.

We present evidence here that all PV sera bind an antigen from epidermis that is very similar to that identified in cultured keratinocytes. We also show that most PV sera bind, in addition, a calcium-sensitive epitope on the PF complex and that the antigen complex identified by PV and PF sera are distinct but share certain biochemical similarities.

\section{Methods}

Sera. Sera from patients with PV, PF (including Brazilian PF), Darier's disease (a nonimmunologically mediated acantholytic disease), bul-

2. The 85,000 -mol-wt polypeptide was previously reported as 80,000 mol wt. Recent additional molecular weight determinations by SDSPAGE approximate the molecular weight as closer to 85,000 . 
lous pemphigiod, and normals were used. Brazilian PF sera were as previously reported (10). PV and PF sera were from patients with clinical and/or histological typical disease. All PV and PF sera demonstrated positive indirect immunofluorescence at titers $\geq \mathbf{4 0}$ with a cell surface pattern on normal human epidermis or monkey esophagus.

Epidermal extraction. Epidermal sheets were obtained by harvesting suction blister roofs raised on the volar forearms of normal volunteers (area $\left.\sim 3 \mathrm{~cm}^{2}\right)(11)$. These sheets were immediately washed with ice-cold Tris-buffered saline (TBS) $(0.15 \mathrm{M} \mathrm{NaCl}$ in $0.01 \mathrm{M}$ Tris- $\mathrm{HCl}$, $\mathrm{pH}$ 7.4) with the addition of $2 \mathrm{mM} \mathrm{CaCl}$. The epidermal sheets were extracted on ice in $2 \mathrm{ml}$ of $0.5 \% \mathrm{NP}-40,2 \mathrm{mM} \mathrm{CaCl}_{2}$, and $1 \mathrm{mM}$ phenylmethylsulfonylfluoride in TBS. The epidermal sheets were crushed with a blunt plastic rod, vortexed for $90 \mathrm{~s}$, and ultrasonicated at setting 6 for $90 \mathrm{~s}$ (microultrasonic cell disrupter, Kontes Co., Vineland, NJ). The extract was centrifuged at $100,000 \mathrm{~g}$ for $1 \mathrm{~h}$ at $4^{\circ} \mathrm{C}$ and the resulting supernatant was preabsorbed with two pellets made from $1 \mathrm{ml}$ each of protein A-bearing Staphylococcus aureus (Pansorbin, Calbiochem-Behring Corp., La Jolla, CA). In certain experiments 2 $\mathrm{mM}$ iodoacetamide was added to the TBS at all steps. The preabsorbed extracted epidermal proteins were then radiolabeled with ${ }^{125} \mathrm{I}$.

Iodination. Extracted epidermal proteins were iodinated using 1 $\mathrm{mCi}$ of $\mathrm{Na}^{125} \mathrm{I}$ (Amersham Corp., Arlington Heights, IL) and iodobeads ( $n$-chloro-benzesulfonamide sodium salt, derivatized uniform, nonporous polystyrene beads) (Pierce Chemical Co., Rockford, IL) (2). 10 beads were washed 3 times with $5 \mathrm{ml}$ TBS and then allowed to dry. Washed and dried beads were charged by adding the radiolabel in 0.5 $\mathrm{ml}$ of TBS for $5 \mathrm{~min}$. The epidermal extract was added to the charged beads for $20 \mathrm{~min}$. Unbound iodine was removed by dialysis at $4^{\circ} \mathrm{C}$ against $0.3 \%$ NP-40 in TBS.

Cell-culture. Normal human epidermal cells from neonatal foreskins were cultured and radiolabeled with ${ }^{14} \mathrm{C}$-amino acids as previously described $(8,12)$.

Immunoprecipitation. Immunoprecipitations were performed as previously described $(2,8,9,12)$ except that in most experiments 2 $\mathrm{mM} \mathrm{CaCl}_{2}$ was added to all buffers and washing solutions. Approximately $20 \times 10^{6} \mathrm{cpm}$ of ${ }^{125}$ I-labeled epidermal extracts or $4 \times 10^{6} \mathrm{cpm}$ of ${ }^{14} \mathrm{C}$-labeled cultured keratinocyte extracts and $10-15 \mu \mathrm{l}$ of sera were used per immunoprecipitation. As usual, immunoprecipitated proteins were only denatured after precipitation and washing by boiling the pellet for $2 \mathrm{~min}$ in $100 \mu \mathrm{l}$ of sample buffer (2\% SDS, $10 \%$ glycerol, and $0.01 \%$ bromphenol blue in $0.0625 \mathrm{M}$ Tris- $\mathrm{HCl}, \mathrm{pH} \mathrm{6.8)}$ and, if reduced, $0.1 \mathrm{M}$ DTT. Precipitated polypeptides were resolved by SDSPAGE or into two dimensions by isoelectric focusing followed by SDS-PAGE as previously described (3). Separated proteins were then visualized by autoradiography using enhancing screens (2).

Diagonal nonreduced-reduced gels. Immunoprecipitated proteins were eluted from protein A without DTT and then separated under nonreducing conditions by SDS-PAGE in 3.4-mm tube gels (13). Tubes were then incubated for $90 \mathrm{~min}$ at room temperature in sample buffer with $10 \mathrm{mM}$ DTT. Each tube was sealed on a slab gel with agarose ( $1 \%$ agarose, $0.1 \%$ SDS, and $2.5 \mathrm{mM}$ DTT in $0.0625 \mathrm{M}$ Tris$\mathrm{HCl}, \mathrm{pH}$ 6.8) and separated in the second dimension under reducing conditions by SDS-PAGE. Proteins were then visualized using autoradiography with enhancing screens.

\section{Results}

Immunoprecipitation of the PV antigen from extracts of normal human epidermis. All PV sera tested precipitated a characteristic complex (PV complex) of polypeptides of $\sim 210,000,130,000$, and 85,000 mol wt in epidermal extracts containing $2 \mathrm{mM}$ calcium (Fig. 1). This complex is different from the complex of proteins (called here PF complex) precipitated by PF sera from epidermal extracts (Fig. 1 and reference 2) of $260,000,160,000,110,000$, and $85,000 \mathrm{~mol} \mathrm{wt}$. All $22 \mathrm{PV}$ sera tested to date have demonstrated this binding, and none

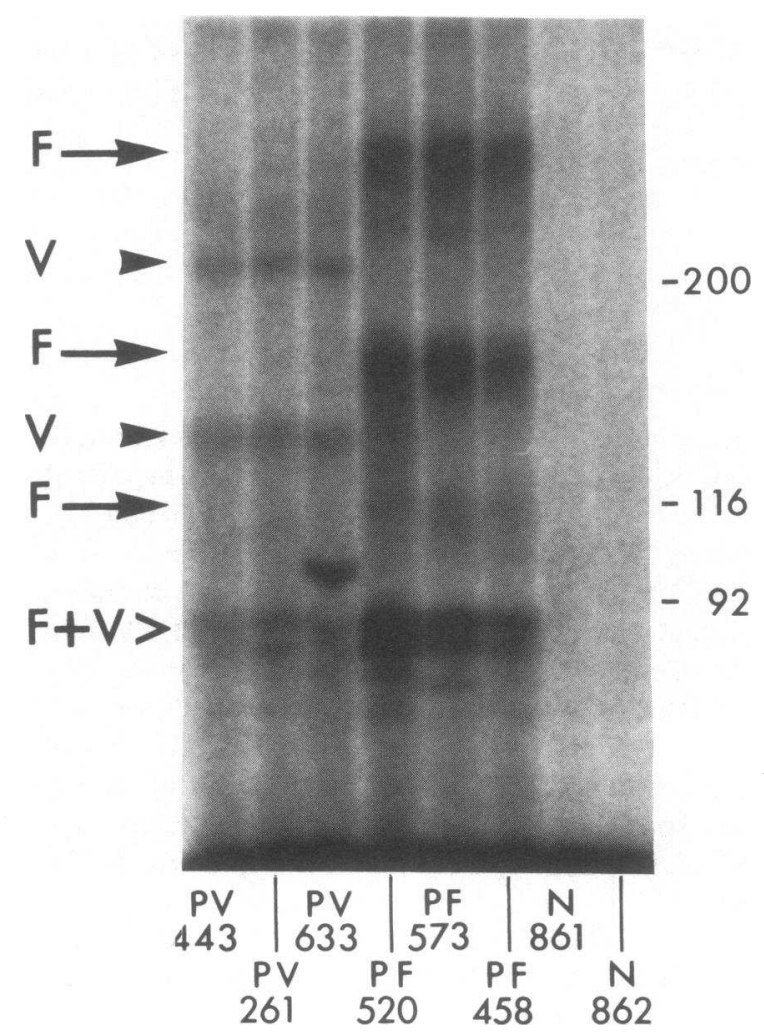

Figure 1. PV antigen extracted from normal human epidermis is a characteristic complex of polypeptides distinct from $\mathrm{PF}$ antigen. ${ }^{125} \mathrm{I}$ labeled extracts of normal human epidermis were immunoprecipitated with PV sera, PF sera, or normal human sera (N). $2 \mathrm{mM} \mathrm{CaCl}_{2}$ was added to the extraction and immunoprecipitation buffers. Immunoprecipitated proteins were reduced, separated by SDS-PAGE, and visualized by autoradiography. PV sera (different numbers corresponding to different patients) precipitated a complex (PV complex) of $210,000,130,000$, and $85,000 \mathrm{~mol}$ wt (marked by the Vs) that was distinct from the complex of polypeptides (PF complex), immunoprecipitated by PF sera, of $260,000,160,000,110,000$, and 85,000 mol wt (marked by the Fs). Normal sera did not precipitate these complexes. The migration of molecular weight standards is indicated by the numbers (molecular weight $\times 10^{-3}$ ) on the right side of the gel.

of 17 PF sera tested have bound the PV complex. Neither the PV nor PF complex was recognized by 28 control sera. Controls included 2 patients with Darier's disease (a nonimmunologically mediated acantholytic disease), 7 patients with bullous pemphigoid (a subepidermal immunologically mediated blistering skin disease), and 19 normal sera.

Although none of the sera from PF patients precipitated the PV complex, 14 of the 22 PV sera tested also precipitated the PF complex when extracts and immunoprecipitations were done in the presence of $2 \mathrm{mM}$ calcium (Fig. 2). 7 of these 14 PV sera were tested on extracts that had calcium removed by chelation with EGTA and all then reacted only with the PV complex, although less intensely than if calcium were present (Fig. 3). These data indicate that some PV sera, in addition to having antibodies to the PV complex, have antibodies to a calcium-sensitive epitope on the PF complex. Similarly, we have previously shown that most PF sera also bind a calciumsensitive epitope on the PF complex (2).

Similarities between the $P V$ and $P F$ antigen complexes. To more closely compare the PV complex with the PF complex, 


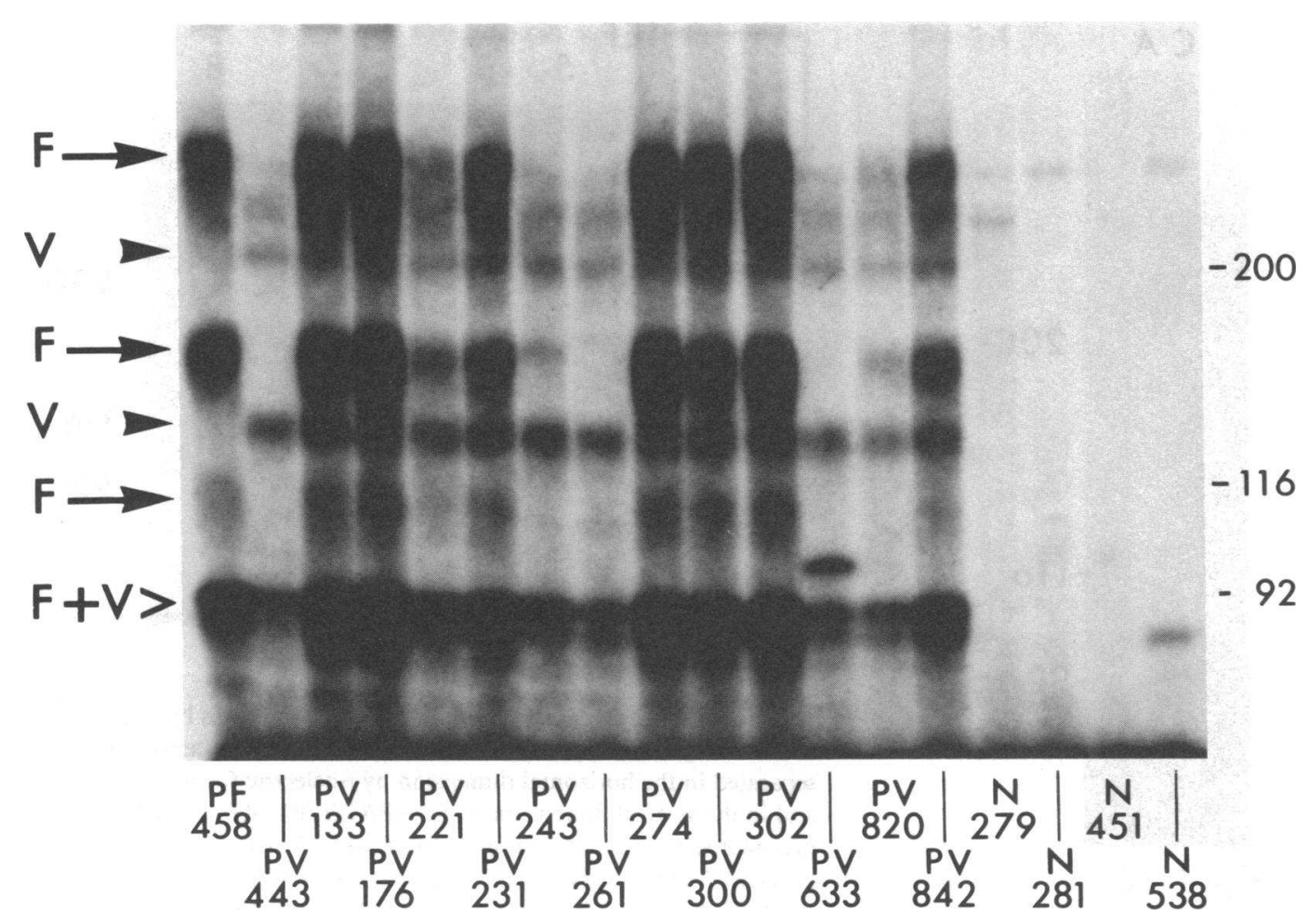

Figure 2. Some PV sera immunoprecipitate both the PV and PF complexes. Immunoprecipitates of extracts of normal human epidermis were performed as in Fig. 1. The first lane is from a patient with $\mathrm{PF}$ and only the PF complex is precipitated (marked by the Fs). All PV sera tested precipitated the PV complex (marked by the Vs), but some also precipitated the PF complex to varying degrees. Normals did not precipitate either complex. N, Normal human sera. we performed two-dimensional gel separation (isoelectric focusing followed by SDS-PAGE) of immunoprecipitated proteins using a PV sera that recognizes both complexes in the presence of calcium (Fig. 4). The 130,000-mol-wt polypeptide from the PV complex has the same isoelectric point as the 160,000-mol-wt polypeptide from the PF complex, as do the PV 210,000- and PF 260,000-mol-wt polypeptides. In addition, the 85,000-mol-wt polypeptide from both complexes comigrate. The same results were obtained when the PF complex, precipitated by PF sera, and the PV complex, precipitated by PV sera, were compared on simultaneously run two-dimensional gels.

Further similarities between PV and PF complexes were revealed by nonreduced-reduced diagonal gels, as discussed below.

Diagonal gels indicate that peptides in both the PV and PF complexes are covalently (disulfide) and noncovalently linked. In two-dimensional diagonal gels, proteins are first separated by SDS-PAGE without reducing agents and then in the second dimension by SDS-PAGE after reduction of disulfide bonds. Proteins without disulfide bonds migrate equally in both dimensions and thus fall on the diagonal. Proteins with interchain disulfide bonds migrate below the diagonal in the second dimension at a point under the diagonal equal to their unreduced molecular weight. Diagonal gels of the PV and PF complexes look remarkably similar (Fig. 5). Some of the 210,000 mol-wt peptide of the PV complex is formed by disulfide linkage of the 130,000 - and 85,000-mol-wt peptides. A similar linkage to form an $\sim 260,000$-mol-wt peptide also occurs between the 160,000 - and the 85,000-mol-wt peptides of the PF complex. There are also some nondisulfide-linked peptides of the complexes present, migrating on the diagonal, which indicates that some peptides are also present in the complex as noncovalently linked peptides. After reduction, much of the
210,000- and 260,000-mol-wt peptides are reduced to their component chains; however, some of the 210,000-mol-wt polypeptide of the PV complex and some of the 260,000 -molwt polypeptide of the PF complex persist on the diagonal. There are several possible explanations for this finding. First, there may be hidden nonreducible disulfide bonds that form between the smaller molecular weight polypeptides of each complex. Second, after disulfide bond formation, other nondisulfide covalent bonds may form. Finally, and less likely, the nonreducible higher molecular weight peptide of each complex may be unrelated to the disulfide-linked peptide, which just coincidently has the same molecular weight.

Some of the disulfide bonds that form are a consequence of the extraction and iodination procedure, which subjects proteins to a more oxidizing environment than in vivo. When iodoacetamide (which blocks free sulfhydryl groups and prevents disulfide bonds from forming) is added to the epidermal extraction, solution more of the 130,000- and 85,000-mol-wt polypeptides of the PV complex migrated on the diagonal in nonreduced-reduced gels, indicating they are nondisulfide linked (data not shown). However, even in the presence of iodoacetamide, some of the peptides are still disulfide linked (and therefore migrate below the diagonal). Similar results with and without iodoacetamide were noted with the PF complex (data not shown).

These data indicate that the 130,000 - and 85,000-mol-wt peptides of the PV complex and the 160,000 - and 85,000 mol-wt polypeptides of the PF complex are present both as covalently and noncovalently linked peptides.

$P V$ antigen is similar in epidermal and cultured keratinocyte extracts. The 130,000- and 85,000-mol-wt peptides immunoprecipitated by PV sera from either cultured keratinocyte or epidermal extracts co-migrate when separated by SDSPAGE (Fig. 6) or into two dimensions by isoelectric focusing 


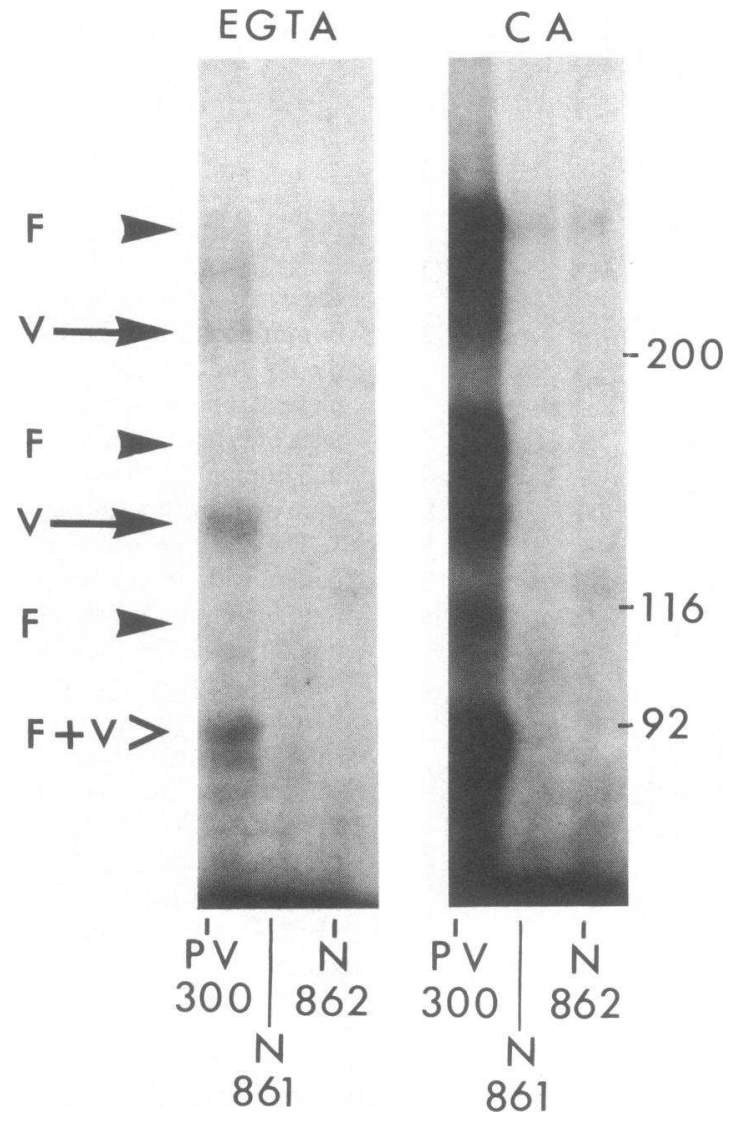

Figure 3. PV serum precipitates the PV and PF complexes in the presence of $2 \mathrm{mM} \mathrm{CaCl}_{2}$, but only the PV complex if calcium is chelated with EGTA. Epidermal proteins were extracted with $0.5 \%$ $\mathrm{NP}-40$ and $2 \mathrm{mM} \mathrm{CaCl}$, then radiolabeled with ${ }^{125}$ I. Immunoprecipitations were then performed either without (lanes labeled CA) or with $4 \mathrm{mM}$ EGTA (lanes labeled EGTA) added to a portion of the extract. Both portions of the extract were precipitated by the same sera. PV 300 precipitated both the PV complex (marked by the Vs) and the PF complex (marked by the Fs) in the presence of $2 \mathrm{mM}$ calcium, but when calcium was removed by chelation with EGTA then only the PV antigen was immunoprecipitated, although less intensely than when calcium was present. Normal human sera $(\mathrm{N})$ do not precipitate either complex.

followed by SDS-PAGE (data not shown). As discussed above, the 210,000 -mol-wt polypeptide seen only in the epidermal extract in these reduced gels may represent covalently linked (either nondisulfide linked or linked by hidden nonreducible disulfide bonds) 130,000- and 85,000-mol-wt peptides, or, less likely, a polypeptide unrelated to the smaller polypeptides of the complex. It has been shown that the 130,000 - and 85,000 mol-wt polypeptides from cultured keratinocytes form disulfide bonds under nonreducing conditions to make a 210,000 mol-wt peptide (9), just as do the 130,000 - and 85,000-mol-wt polypeptides extracted from normal human epidermis (Fig. 5). These data demonstrate the similarity of the PV antigen isolated from cultured keratinocytes to that isolated from normal epidermis.

\section{Discussion}

All PV sera tested have antibodies to a characteristic complex of polypeptides extracted from normal human epidermis, two

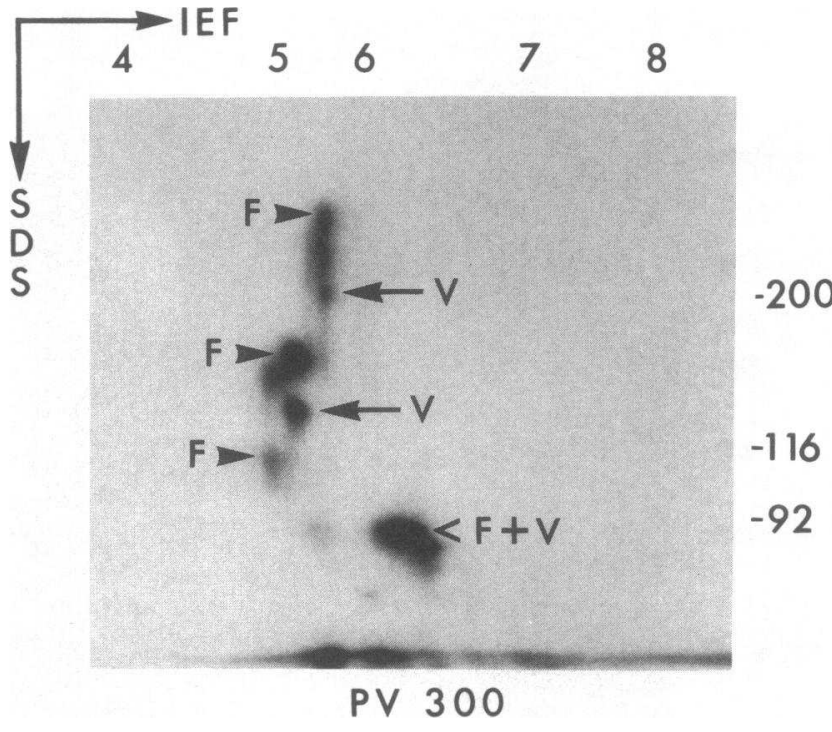

Figure 4. Comparison of PV and PF antigen complexes by two-dimensional gel electrophoresis. Immunoprecipitated proteins were separated in the horizontal dimension by isoelectric focusing (IEF), and in the vertical dimension by SDS-PAGE. PV 300 is a serum that precipitates both the PV complex (marked by Vs) and the PF complex (marked by Fs) (see Fig. 3). The 210,000 and 260,000 as well as the 130,000 and 160,000 -mol-wt peptides of both complexes have identical isoelectric points. In addition, the 85,000 -mol-wt peptide (marked by the $F+V$ ) of both complexes co-migrate. The numbers at the top of the gel indicate the $\mathrm{pH}$ gradient of the isoelectric focusing gel. The numbers to the right of the gel are the migration of known molecular weight standards (molecular weight $\times 10^{-3}$ ).

peptides of 130,000 and $85,000 \mathrm{~mol}$ wt that are capable of forming disulfide and possibly other covalent bonds. About two-thirds of the PV sera tested also have antibodies to a calcium-sensitive epitope on the PF complex. The following observations suggest that the PV and PF complexes are separate and distinct, and that some PV patients have separate antibody systems against each: $(a)$ all PF sera tested bind only the PF complex (reference 2 and Fig. 1); (b) some PV sera precipitate only the PV complex (Figs. 1 and 2); and (c) those PV sera that precipitate both complexes bind only the PV complex in the absence of calcium (Fig. 3).

The PV and PF antigen complexes, although distinct, have certain similarities. The 130,000-mol-wt peptide of the PV complex and the 160,000-mol-wt peptide of the PF complex have the same isoelectric point and both bind the same 85,000 -mol-wt peptide through disulfide as well as noncovalent bonds.

No laboratory or clinical differences were noted among PV patients with sera that did or did not also bind the PF complex. It appears that if antibodies are present to the PV complex, suprabasilar acantholysis occurs whether or not antibodies are also present against the PF complex. Although both PF and PV antigens are usually present in a similar distribution by immunofluorescence (1) they are distinct complexes and may serve to mediate adhesion at different levels in the epidermis. In fact, in some cases, PF antibodies have been reported to be located in the superficial epidermis only $(14,15)$.

The PV antigen which we have previously characterized from extracts of keratinocyte cultures $(8,9)$ is similar to the 

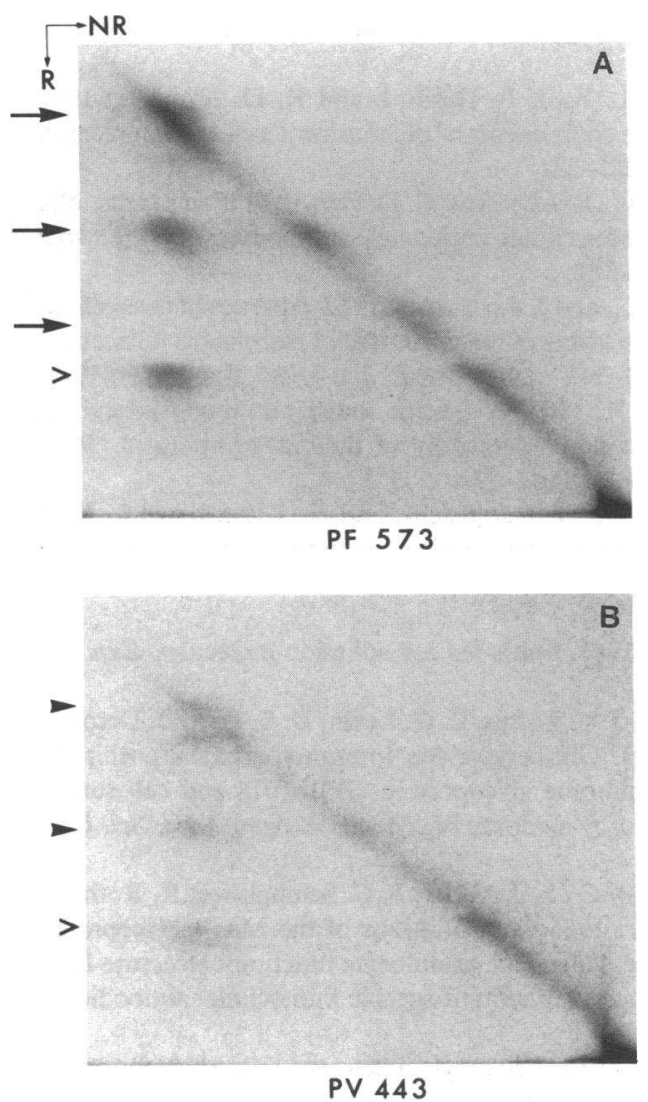

Figure 5. The peptides of the PV and PF complexes are both disulfide linked and noncovalently associated. Autoradiogram of ${ }^{125} \mathrm{I}$-labeled epidermal proteins immunoprecipitated either with PF serum or with PV serum and separated by diagonal gels into two dimensions. Proteins were first separated in the horizontal direction by SDS-PAGE without reducing agents (NR) and then in the vertical direction by SDS-PAGE after reduction of disulfide bonds ( $R$ ). Proteins without disulfide bonds migrate equally in both dimensions and thus fall on the diagonal. Proteins with interchain disulfide bonds migrate below the point on the diagonal corresponding to their unreduced molecular weight. Note that in $A$ the 260,000-mol-wt peptide of the PF complex (marked by the top arrow) is at least partially formed by disulfide-linked 160,000 (marked by the middle arrow)and 85,000 (marked by the open arrowhead)-mol-wt peptides. The 110,000-mol-wt peptide (marked by the lower arrow) and some of the 160,000- and 85,000-mol-wt peptides are also present on the diagonal, indicating they are also noncovalently associated in the complex. A similar result is seen in the PV complex $(B)$. The 210,000mol-wt peptide (top closed arrowhead) is at least partially formed by disulfide-linked 130,000 (bottom closed arrowhead)- and 85,000 (bottom open arrowhead)-mol-wt peptides. However, some 130,000 and 85,000-mol-wt peptides are present on the diagonal, indicating they are noncovalently associated as well.

antigen we have described here extracted from human epidermis. We suspect that our inability to detect PV antigen by immunoblots of extracts of human epidermis (9) relates to the fact that antigen is fully denatured before being exposed to antibody in the immunoblot procedure. In contrast, in the immunoprecipitation procedure used here (and in the previous cell culture study) the antigen is not fully denatured until after it has been precipitated by the antibody. We have also

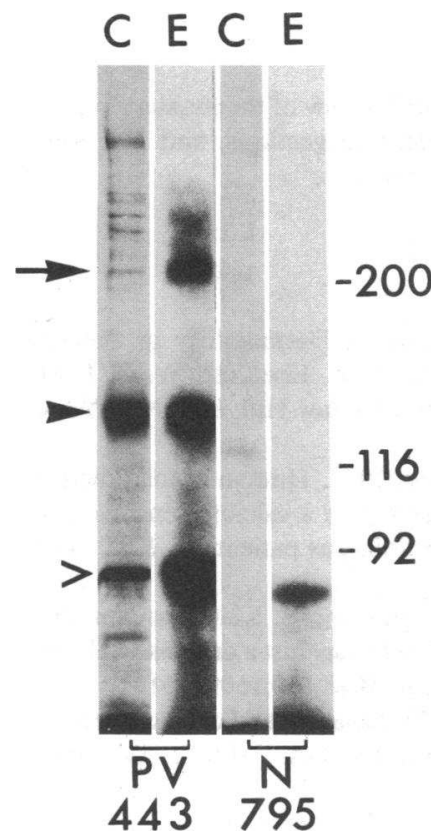

Figure 6. Comparison of PV antigen extracted from cultured keratinocytes and epidermis. Autoradiogram of SDS-PAGE of radiolabeled immunoprecipitated protein extracted from either cultured keratinocytes $(C)$ or epidermis (E). The 130,000- (closed arrowhead) and the 85,000 (open arrowhead)-mol-wt peptides precipitated by PV sera from extracts of both epidermal and cultured keratinocytes co-migrate. The 210,000-mol-wt peptide (arrow) is present in this reduced gel only in the epidermal extract. The band present in the last lane (immunoprecipitation of the epidermal extract with normal human serum) migrates slightly faster than the 85,000 mol-wt peptide, and also does not co-migrate with the 85,000 -mol-wt peptide on two-dimensional gels (data not shown).

noted this problem of antibody not binding denatured antigen on immunoblots with the majority of PF sera $(3,9,10)$ and with some pemphigoid and epidermolysis bullosa acquisita sera $(16,17)$. However, note that Peterson and Wuepper (6) were able to detect a PV antigen by immunoblotting if they concentrated the human PV sera that they used. This PV antigen had a molecular weight of 33,000 on SDS-PAGE. How this low molecular weight antigen relates to the PV complex described here is unclear and awaits further elucidation.

As suggested by Jones et al. (7), PV antigen may be a member of a large group of so-called cell adhesion molecules which includes molecules such as L-CAM, N-CAM, and uvomorulin (18). PF antigen might also prove to be in this category. It is also possible that the PV and PF complexes may belong to a family of molecules called cytoadhesions, or integrins, which are important in cell-cell and cell-substrate adhesion and include molecules such as Mac-1, LFA-1, and platelet glycoproteins (GIIb/IIIa (19-22). Integrins are comprised of noncovalently linked heterodimers with a small component of 95,000-130,000 mol wt and a large component with molecular weight between 130,000 and 210,000 . The possible relationship of the PV and PF antigens to these molecular families awaits further studies.

Whatever the relationship, if any, of PV and PF antigens to these other molecular families, these antigens are clearly related to each other, and may prove to be important molecules in epidermal cell adhesion. Exactly how autoantibodies from these patients interact with these molecular complexes to cause loss of cell adhesion, whether directly by interfering with their function or indirectly through protease release by keratinocytes $(23,24)$, complement activation (25), or combinations of these processes, merits further study. In any case, the autoantibodies from these patients can now be used to absolutely distinguish PV and PF at a molecular level. 


\section{Acknowledgments}

We thank Dr. Stephen Katz for critical review of the manuscript, Dr. Richard Klausner for many excellent suggestions, and Ms. Vera Klaus-Kovtun for able technical assistance.

\section{References}

1. Jordon, R. E. 1987. Pemphigus. In Dermatology in General Medicine. 2nd ed. T. B. Fitzpatrick, A. Z. Eisen, K. Wolff, I. M. Freedberg, and K. F. Austen, editors. McGraw-Hill, Inc., New York. 571-579.

2. Eyre, R. W., and J. R. Stanley. 1987. Human autoantibodies against a desmosomal protein complex with a calcium-sensitive epitope are characteristic of pemphigus foliaceus patients. J. Exp. Med. 165:1719-1724.

3. Koulu, L., A. Kusumi, M. S. Steinberg, V. Klaus-Kovtun, and J. R. Stanley. 1984. Human autoantibodies against a desmosomal core protein in pemphigus foliaceus. J. Exp. Med. 160:1509-1518.

4. Shu, S., and E. H. Beutner. 1973. Isolation and characterization of antigens reactive with pemphigus antibodies. J. Invest. Dermatol. 61:270-276.

5. Diaz, L. A., H. Patel, and N. J. Calvanico. 1980. Isolation of pemphigus antigen from human saliva. J. Immunol. 124:760-765.

6. Peterson, L. L., and K. D. Wuepper. 1984. Isolation and purification of a pemphigus vulgaris antigen from human epidermis. J. Clin. Invest. 73:1113-1120.

7. Jones, J. C. R., K. M. Yokoo, and R. D. Goldman. 1986. Further analysis of pemphigus autoantibodies and their use in studies on the heterogeneity, structure, and function of desmosomes. J. Cell Biol. 102:1109-1117.

8. Stanley, J. R., M. Yaar, P. Hawley-Nelson, and S. I. Katz. 1982. Pemphigus antibodies identify a cell surface glycoprotein synthesized by human and mouse keratinocytes. J. Clin. Invest. 70:281-288.

9. Stanley, J. R., L. Koulu, and C. Thivolet. 1984. Distinction between epidermal antigens binding pemphigus vulgaris and pemphigus foliaceus autoantibodies. J. Clin. Invest. 74:313-320.

10. Stanley, J. R., V. Klaus-Kovtun, and S. A. P. Sampaio. 1986. Antigenic specificity of fogo selvagem autoantibodies is similar to North American pemphigus foliaceus and distinct from pemphigus vulgaris autoantibodies. J. Invest. Dermatol. 87:197-201.

11. Kiistala, U. 1968. Suction blister device for separation of viable epidermis from dermis. J. Invest. Dermatol. 50:129-137.

12. Stanley, J. R., P. Hawley-Nelson, S. H. Yuspa, E. M. Shevach, and S. I. Katz. 1981. Characterization of bullous pemphigoid antigen: a unique basement membrane protein of stratified squamous epithelia. Cell. 24:897-903.

13. Samelson, L. E., J. B. Harford, and R. D. Klausner. 1985. Identification of the components of the murine $\mathrm{T}$ cell antigen receptor complex. Cell. 43:223-231.

14. Bystryn, J. C., E. Abel, and C. DeFeo. 1974. Pemphigus foliaceus. Subcorneal intercellular antibodies of unique specificity. Arch. Dermatol. 110:857-861.

15. Bystryn, J. C., and J. Rodriquez. 1978. Absence of intercellular antigens in the deep layers of the epidermis in pemphigus foliaceus. $J$. Clin. Invest. 61:339-348.

16. Stanley, J. R. 1985. A specific antigen-antibody interaction triggers the cellular pathophysiology of bullous pemphigoid. $\mathrm{Br}$. J. Dermatol. 113(Suppl. 28):67-73.

17. Stanley, J. R., N. Rubinstein, and V. Klaus-Kovtun. 1985. Epidermolysis bullosa acquisita antigen is synthesized by both human keratinocytes and human dermal fibroblasts. J. Invest. Dermatol. 85:542-545.

18. Obrink, B. 1986. Epithelial cell adhesion molecules. Exp. Cell Res. 163:1-21.

19. Plow, E. F., J. C. Loftus, E. G. Levin, D. S. Fair, D. Dixon, J. Forsyth, and M. H. Ginsberg. 1986. Immunologic relationship between platelet membrane glycoprotein GPIIb/IIIa and cell surface molecules expressed by a variety of cells. Proc. Natl. Acad. Sci. USA. 83:6002-6006.

20. Anderson, D. C., L. J. Miller, F. C. Schmalstieg, R. Rothlein, and T. A. Springer. 1986. Contributions of the Mac-1 glycoprotein family to adherence-dependent granulocyte functions: structure-function assesments employing subunit-specific monoclonal antibodies. $J$. Immunol. 137:15-27.

21. Gallin, J. I. 1985. Leukocyte adherence-related glycoproteins LFA-1, Mo-1 and p150,95: a new group of monoclonal antibodies, a new disease, and a possible opportunity to understand the molecular basis of leukocyte adherence. J. Infec. Dis. 152:661-664.

22. Hynes, R. O. 1987. Integrins: a family of cell surface receptors. Cell. 48:549-554.

23. Schiltz, J. R., B. Michel, and R. Papay. 1978. Pemphigus antibody interaction with human epidermal cells in culture. J. Clin. Invest. 62:778-788.

24. Hashimoto, K., K. M. Shafran, P. S. Webber, G. S. Lazarus, and K. H. Singer. 1983. Anti-cell surface pemphigus autoantibody stimulates plasminogen activator and activity of human epidermal cells. J. Exp. Med. 157:259-272.

25. Kawana, S., W. D. Geoghegan, and R. E. Jordon. 1985. Complement fixation by pemphigus antibody. II. Complement enhanced detachment of epidermal cells. Clin. Exp. Immunol. 61:517-525. 